\title{
Daniel Mercure, Mircea Vultur, La signification du travail. Nouveau modèle productif et ethos du travail au Québec
}

Québec, Presses de l'Université Laval, coll. "Sociologie contemporaine », 2010

\section{Léa Lima}

\section{OpenEdition}

\section{Journals}

Édition électronique

URL : https://journals.openedition.org/travailemploi/5687

DOI : $10.4000 /$ travailemploi.5687

ISSN : 1775-416X

\section{Éditeur}

DARES - Ministère du Travail

Édition imprimée

Date de publication : 15 juin 2012

Pagination : 98-99

ISSN : 0224-4365

\section{Référence électronique}

Léa Lima, "Daniel Mercure, Mircea Vultur, La signification du travail. Nouveau modèle productif et ethos du travail au Québec », Travail et Emploi [En ligne], 130 | avril-juin 2012, mis en ligne le 01 décembre 2012, consulté le 30 juillet 2021. URL : http://journals.openedition.org/travailemploi/5687 ; DOI https://doi.org/10.4000/travailemploi.5687

Ce document a été généré automatiquement le 30 juillet 2021.

(C) Direction de l'animation de la recherche, des études et des statistiques (Dares) 


\section{Daniel Mercure, Mircea Vultur, La signification du travail. Nouveau modèle productif et ethos du travail au Québec}

Québec, Presses de l'Université Laval, coll. « Sociologie contemporaine », 2010

\section{Léa Lima}

\section{RÉFÉRENCE}

Daniel Mercure, Mircea Vultur, La signification du travail. Nouveau modèle productif et ethos du travail au Québec, Québec, Presses de l'Université Laval, coll. « Sociologie contemporaine », 2010, $290 \mathrm{p}$.

1 Cet ouvrage éclaire la question de la « valeur du travail » à partir d'une vaste enquête réalisée au Québec en 2007. L'objet du livre est de relire cette question classique en sociologie du travail à l'aune des nouveaux discours managériaux qui redessinent le rapport du travailleur à l'entreprise. Daniel Mercure et Mircea Vultur ont cherché à saisir les effets de la transformation du travail et des normes managériales qui l'organisent sur le rapport au travail de la population active québécoise. Face aux diverses injonctions à l'individuation dans le travail et à la flexibilité des carrières, comment réagissent les individus au plus profond de leurs aspirations? Cette interrogation est déconstruite en de multiples indicateurs qui produisent à la fois la complexité et la finesse des résultats. Partant des résultats empiriques concernant le Québec, l'ambition des auteurs est de se placer à un niveau d'analyse plus global, en cherchant à sentir le vent du nouvel esprit du capitalisme ${ }^{1}$ qui souffle sur les travailleurs et la manière dont ces derniers le traduisent dans leurs opinions. 
2 Une enquête par questionnaire auprès de 1000 personnes permet aux auteurs de répartir la population active en six « formes générales d'orientation au travail » à partir d'indicateurs de la centralité du travail (forte, moyenne, faible) et de la finalité principale que les individus lui assignent : externe (le travail comme moyen) ou interne (le travail comme fin en soi ou l'expérience vécue du travail). Puis chacune de ces «formes » est décrite du point de vue des valeurs, attitudes, et croyances attachées au travail dans une perspective plus compréhensive à l'aide d'entretiens semi-directifs (au nombre de cinquante-deux) au cours desquels les individus étaient interrogés sur leur parcours professionnel, le sens donné au travail, leur perception des attentes des employeurs ainsi que l'évaluation de la qualité de leur emploi. Les six formes repérées précédemment ont révélé alors autant d'ethos du travail (ethos de l'autarcie, ethos de la professionnalité, ethos utilitariste, ethos égotéliste, ethos de la résignation, et ethos de l'harmonie).

3 L'enquête conforte tout d'abord des résultats portant sur d'autres aires culturelles, attestant de l'importance que les individus accordent encore au travail et ce d'autant plus, comme le montrait l'enquête sur le bonheur et le travail en France ${ }^{2}$, que l'emploi vient à manquer. Elle met aussi en lumière la quête généralisée d'un équilibre entre vie professionnelle et vie hors travail (vie de famille au premier chef). Les auteurs vont donc à l'encontre des thèses de la «fin du travail » qu'elles soient entendues au sens large de la dévalorisation absolue du travail, ou dans un sens plus étroit, comme instrumentalisation du travail. À travers la "valeur travail », une majorité d'individus (58\% des personnes interrogées) exprime son attachement à l'expérience concrète du travail, que ce soit pour le contenu des tâches, l'activité globale ou le cadre relationnel, validant les thèses post-matérialistes sur la recherche d'épanouissement personnel dans le travail ${ }^{3}$. Sans surprise, ce sont ceux qui sont au bas de l'échelle sociale (en termes de ressources et de niveau de formation) qui donnent le plus souvent une finalité économique au travail. Les chercheurs soulignent aussi une assez forte adhésion aux nouvelles normes managériales prônant l'individualisation des carrières, la responsabilisation des travailleurs dans la gestion de leur travail et de leur avenir professionnel et, dans une moindre mesure, l'investissement personnel dans le travail ainsi que la flexibilisation dans la gestion des différentes temporalités. En effet, l'exigence de se rendre disponible en dehors des heures de travail (plus de la moitié d'opinions défavorables) et d'investir son temps personnel pour la formation (un tiers d'opinions défavorables) entre en conflit avec le désir des individus de conserver un certain équilibre entre vie professionnelle et vie privée.

4 L'adhésion à ce nouveau schème de la production flexible est consubstantielle à des changements culturels bien plus profonds. Le livre montre bien que le nouvel esprit du capitalisme s'accommode d'ethos aux orientations relativement différentes : celui qui construit son identité par le travail et pour le travail et celui qui poursuit son développement personnel et l'affirmation de son individualité dans toutes les sphères de la vie, dont celle du travail. Représentant un tiers de la population active au Québec, ce dernier type est non seulement le plus répandu, mais aussi, selon les auteurs, porteur de l'avenir du travail. Non totalement soumises aux exigences de l'entreprise puisque revendiquant un fort équilibre entre vie personnelle et vie au travail, l'exigence d'autonomie et la quête de réalisation de soi peuvent néanmoins être facilement mobilisées sur le lieu de travail dans l'esprit managérial libéral. Au contraire, d'autres individus dont les valeurs expriment un changement culturel 
pourtant important demeurent à la marge du nouveau système productif : dans le pôle négatif, l'ethos de la résignation, produit de la précarisation de l'emploi, convertit l'activité professionnelle en simple moyen de subsistance et marque une forte distance par rapport à la doxa managériale; l'ethos de l'harmonie en est son double positif: le travail et l'entreprise sont mis à distance non par dépit mais par choix.

On pourra discuter le recours à l'outillage conceptuel de l'ethos, qui tend à essentialiser les positions des individus présentées de manière peut-être trop figée. En mettant l'accent sur les caractéristiques socioprofessionnelles des personnes au temps $t$ sans rendre compte des trajectoires du point de vue des positions sociales et des statuts d'emploi, les auteurs s'empêchent de comprendre les processus par lesquels se forgent les valeurs et les opinions des personnes. Alors que l'avènement du post-fordisme et de son idéologie du travail peut être daté, il est difficile avec cette enquête de trancher sur un hypothétique effet de génération en termes de construction identitaire puisqu'on manque de données comparatives dans le temps, que ce soit dans une optique longitudinale à travers l'interrogation répétée d'un panel d'individus pris à des moments différents de la socialisation au travail et/ou dans une approche plus simplement diachronique en réitérant cette enquête transversale à intervalle plus régulier. Les auteurs fournissent des interprétations qui nourrissent aussi bien l'hypothèse d'un effet du cycle de vie, lorsqu'ils évoquent les caractéristiques en termes d'âge et de situation de famille des individus porteurs de telle ou telle "forme ", que celle d'un effet de période lorsqu'ils insistent en conclusion sur les transformations des valeurs et des attentes. Comme nous l'indiquent les chercheurs, l'enquête a été réalisée au point paroxystique d'un cycle de croissance économique et de création d'emplois ; il est donc difficile d'en tirer des conclusions en termes de changement social.

En outre si un chapitre est accordé à l'évolution de la population active et en emploi au Québec, la spécificité institutionnelle et politique du cas québécois est largement passée sous silence dans l'ouvrage: il n'est par exemple pas fait mention des politiques sociales ou des réformes du marché du travail qui pourraient avoir un impact sur le rapport au travail, alors que le modèle social, considéré comme plus protecteur et progressiste que dans le reste du continent nord-américain, est (était?) un marqueur fort de l'identité québécoise. Il n'est pas non plus fait référence à la situation géopolitique particulière du Québec et de ses habitants, et à la distanciation qu'ils peuvent éprouver vis-à-vis du monde anglophone singulièrement dans leur rapport au travail. Il faudrait, pour distinguer dans les résultats ce qui découle d'un mouvement plus global d'internationalisation des normes de management et ce qui relève de l'histoire culturelle plus singulière de cet îlot francophone, disposer d'éléments de contextualisation complémentaires. Reste que, de par la richesse des matériaux, ce livre intéressera particulièrement les sociologues en quête de données actualisées sur l'état du rapport au travail aujourd'hui ou des gestionnaires qui cherchent à évaluer l'emprise des nouveaux modèles de gestion des ressources humaines sur les salariés. 


\section{NOTES}

1. Boltanski L., Chiapello E., Le nouvel esprit du capitalisme, Paris, Gallimard, 1999.

2. Baudelot C., Gollac M., Travailler pour être heureux? Le bonheur et le travail en France, Paris, Fayard, 2003.

3. Inglehart R., The Silent Revolution, Londres, MacMillan, 1977.

\section{AUTEURS}

\section{LÉA LIMA}

Conservatoire national des arts et métiers, LISE 